\title{
Clinical Evaluation of Laparoscopic Cholecystectomy in the Treatment of Acute Cholecystitis in the Elderly
}

\author{
FEI GUO, ANNA BAO, HAO ZHANG AND SEN LIN CHEN*
}

Department of General Surgery, Shanghai Chang Hai Hospital, Hongkou, Shanghai 200233, China

Guo et al.: Laparoscopic Cholecystectomy Efficacy in the Treatment of Acute Cholecystitis

\begin{abstract}
To evaluate the efficacy of laparoscopic cholecystectomy in the treatment of senile acute cholecystitis. A total of 96 elderly patients with acute cholecystitis admitted to our hospital from January 2014 to January 2019 were selected and all the patients were divided into groups according to their treatment timing. Patients within $3 \mathrm{~d}$ from the onset of the disease to the operation time were selected as Group A. Patients with an onset time of $3 \mathrm{~d}$ to $3 \mathrm{w}$ of surgery were treated as Group B, while patients with an onset time of more than $3 \mathrm{w}$ of surgery were treated as Group C. The operative time, intraoperative blood loss, postoperative intestinal function recovery time and hospital stay time were compared among the groups. Postoperative complications such as bleeding, pulmonary infection, abdominal infection, bile leakage and intestinal fistula, deep vein thrombosis and myocardial infarction were compared among all groups. Compared with Group A, there were no significant differences in intraoperative blood loss, operative time, postoperative bowel function recovery and length of hospital stay in Group $B(p>0.05)$. Compared with group $\mathrm{A}$ and $\mathrm{B}$, patients in Group $\mathrm{C}$ had higher operative time, intraoperative blood loss, postoperative intestinal function recovery and hospital stay $(\mathbf{p}<\mathbf{0 . 0 5})$. After surgery, there were no significant differences in bleeding, pulmonary infection, abdominal infection, bile leakage and intestinal fistula, deep vein thrombosis, or complications of myocardial infarction among the groups $(p>0.05)$. Early laparoscopic cholecystectomy can reduce intraoperative blood loss, operation time, hospital stay and recovery time of intestinal function in elderly patients with acute cholecystitis.
\end{abstract}

Key words: Laparoscopic cholecystectomy, cholecystitis, chronic obstructive pulmonary disease

Acute cholecystitis is inflammation of the gallbladder caused by obstruction of the cystic duct and bacterial invasion $^{[1]}$. The main clinical manifestations are pain in the upper right abdomen, which may also be accompanied by fever, nausea and vomiting and a small number of patients will also have symptoms of jaundice. Acute cholecystitis is a relatively common disease in clinic, with a high incidence, second only to acute appendicitis. For the treatment of acute cholecystitis, surgical treatment is often adopted in clinical practice. However, for elderly patients, because they are often accompanied by a variety of chronic diseases, the function of important organs is reduced and incomplete and they cannot tolerate the trauma caused by surgery, so choose one. This kind of treatment method suitable for elderly patients with acute cholecystitis is particularly important. Laparoscopic cholecystectomy (LC) has a small incision, less trauma to the patient and quicker recovery after surgery. It has become the first choice for the treatment of acute cholecystitis ${ }^{[2]}$. However, the efficacy and safety of LC for the treatment of acute cholecystitis in the elderly have not been widely recognized clinically. In recent years, with the continuous development of medical minimally invasive techniques and the maturity of laparoscopic surgery techniques, LC has gradually begun to be used in the treatment of elderly acute cholecystitis and the efficacy of elderly cholecystitis has also been significantly improved. This article analyzes and evaluates the efficacy of LC in the treatment of acute cholecystitis in the elderly. A total of 96 elderly patients with acute cholecystitis admitted to our hospital from January 2014 to January 2019 were selected, including 56 males and 40 females, aged 60-85 y and the time from onset to surgery was 6 h- $45 \mathrm{~d}$; clinical manifestations There were 88 cases of right upper abdomen pain, 10 cases of

*Address for correspondence

E-mail: Chensl666@163.com 
fever, 28 cases of nausea and vomiting and 10 cases of skin and sclera. Among them, 40 cases had other diseases, including 12 cases of diabetes, 8 cases of essential hypertension, 10 cases of chronic obstructive pulmonary disease and 10 cases of other diseases. All patients were divided into early operation group (within $3 \mathrm{~d}$ ), delayed operation group ( $3 \mathrm{~d}$ to $3 \mathrm{w}$ ) and elective operation group (over $3 \mathrm{w}$ ) according to the time from onset to operation, with 32 cases in each group. All patients had perfect preoperative preparations, except for choledocholithiasis by routine Magnetic resonance cholangio-pancreatography (MRCP) examination. The patients and their family members were informed of this study and they had all signed an informed consent form. Inclusion criteria-Complete clinical data; No history of abdominal surgery; Can tolerate surgery; No history of mental illness; No severe liver or kidney or other organ insufficiency. Exclusion criteria-Those with coagulation dysfunction and bleeding tendency; Severe heart and lung insufficiency; Severe autoimmune diseases; Acute cholangitis; Treatment compliance poor; Those with severe tumors or combined cancers. Before performing LC, the patient will be intubated with general anesthesia, $\mathrm{CO}_{2}$ pneumoperitoneum is established to maintain proper pressure and the "threehole method" is used to insert laparoscope to explore the abdominal cavity. If the gallbladder tension is too large during the operation, it will be reduced first. Pressure, blunt, sharp combined anatomy of the Calot triangle to expose the cystic duct and common bile duct and remove the gallbladder. The gallbladder bed is treated with electrocoagulation to stop bleeding and the surgical area is flushed and sucked clean. If the patient's inflammation is more serious, an abdominal drainage tube should be placed, or for more severe cases, intraoperative frozen section pathology should be performed if necessary and anti-infection treatment should be actively carried out. After the operation, the patient's vital signs were monitored and the drainage condition was observed. Surgery status-The operation time, intraoperative blood loss, postoperative bowel function recovery time and hospital stay were calculated for each group of patients. Complications-Postoperative bleeding, pulmonary infection, incision and abdominal cavity infection, bile leakage and intestinal fistula, deep vein thrombosis and myocardial infarction were counted in each group of patients. Statistical package for the social sciences (SPSS) 22.0 statistical software was used to analyze the data. Among them, the measurement data conforming to the normal distribution are represented by the mean \pm standard deviation $(\overline{\mathrm{x}} \pm \mathrm{s})$, the comparison between groups is by $t$ test; the count data is represented by the number of cases (n) or percentage (\%) and the data comparison is by the $\chi^{2}$ test. When $\mathrm{p}<0.05$, the difference is statistically significant. Comparing the gender, average age, course of body mass index (BMI), clinical manifestations, and other types of diseases in each group of patients, there were no significant differences and no statistical significance $(p>0.05)$. Comparability between groups shown in Table 1. Comparing the operation conditions of each group, the results showed that there was no significant difference in the operation time, intraoperative blood loss, postoperative bowel function recovery time and hospital stay in Group A and Group $\mathrm{B}$ and it was not statistically significant $(\mathrm{p}>0.05)$; However, the operation time, intraoperative blood loss, postoperative bowel function recovery time and hospital stay in Group C were significantly higher than those in Groups A and B. The differences were statistically significant $(p<0.05)$. shown in Table 2. Comparing the postoperative complications of bleeding, lung infection, incision and abdominal cavity infection, bile leakage and intestinal fistula, deep vein thrombosis and myocardial infarction in each group of patients, it was found that there were no significant differences in the complications of patients in each group. It is not statistically shown in Table 3. Acute cholecystitis mainly occurs with local edema, inflammation, hemorrhage and other histopathological changes, which can easily cause adhesion of the gallbladder and surrounding tissues ${ }^{[3]}$. Acute onset and rapid development of the disease. If acute cholecystitis is not controlled in time and effectively, it will often cause cystic duct obstruction. At this time, the patient's condition worsens and will show high fever, severe right upper abdominal pain, gallbladder effusion and empyema, if the condition is further in development, then gallbladder necrosis, perforation, gallbladder duodenal fistula, jejunal fistula, gallbladder biliary fistula, complicated acute pancreatitis and other symptoms will appear, which will threaten the life of patients ${ }^{[4]}$. As for elderly patients, as they grow older, their physiological functions decline, their immunity is reduced, the body's emergency response time to inflammation is long, symptoms and signs are lighter than pathological changes and are often associated with various chronic diseases. The patient's condition progresses faster and the fatality rate is higher ${ }^{[5]}$. Clinically, the traditional treatment of acute cholecystitis is mainly open surgery for cholecystectomy or gallbladder fistula, which brings greater trauma to the 
patient. However, the elderly patients have poor physical fitness, slow recovery and prone to complications. It is suitable for the treatment of acute cholecystitis in the elderly. In recent years, with the advancement and development of minimally invasive technology, LC has been used clinically to replace the previous open surgery and become the standard surgical procedure for the treatment of acute cholecystitis. It has been widely used in the treatment of elderly patients with acute cholecystitis. The timing of LC surgery will have a certain impact on the patient's therapeutic effect. Although the tissue is fragile within $72 \mathrm{~h}$ of the patient's onset, the patient's anatomical level is clear at this time, the adhesions around the gallbladder are not dense and the separation is easier, so the best effect of LC is within $72 \mathrm{~h}$ of the patient's onset. However, studies have shown that only focusing on the timing of surgery is not objective enough for the success of the operation. The key to the success of LC surgery is the correct handling of the Calot triangle anatomy. If it is not handled properly, it may cause damage to the patient's extrahepatic bile duct. When performing LC surgery, you should pay attention to carefully observe the "threetube" structural relationship, that is, the relationship between the cystic duct, the common bile duct and the gallbladder blood vessels, close to the gallbladder and use a suction device to "push, pull and suck" during the operation to help reveal the Calot triangle of the gallbladder and anatomy is essential ${ }^{[6]}$. When the gallbladder bed oozes too much blood, it can be compressed with hot saline gauze and the gallbladder bed can be treated with electrocoagulation to stop the bleeding. The operator should take precautions for any accidents before the operation to ensure the smooth progress of the operation ${ }^{[7]}$. Scholars such as Umemura
$\mathrm{A}^{[8]}$ found that LC is not only effective in the treatment of patients with acute cholecystitis, but also can improve the immune function of patients. In the study of Kowalewski and other scholars ${ }^{[9]}$, it was found that LC can significantly improve the condition of acute cholecystitis in the elderly and improve the quality of life. In the study of Lockhart and other scholars ${ }^{[10]}$, it was found that for patients with acute cholecystitis, the sooner the operation is performed, the better the effect is. In the research of Matsui et al. ${ }^{[11]}$ scholars also found that performing LC at each stage of the onset of patients had no significant effect on the incidence of intraoperative complications. In the above studies, it was found that the intraoperative blood loss, hospitalization time, operation time and intraoperative bowel function recovery time of Group $\mathrm{C}$ patients were significantly higher than those of Groups A and B, which indicates that early laparoscopic surgery, can benefit patients with acute cholecystitis of postoperative recovery. However, in the above mentioned study, it was also found that there were no significant differences in the postoperative complications of the three groups of patients, which indicates that the choice of surgical timing has no significant effect on the incidence of intraoperative complications. This is consistent with the results of many scholars mentioned above and the incidence of intraoperative complications in each group of patients is low. This may be related to LC's ability to effectively distinguish the cystic duct and gallbladder blood vessels. This is similar to the results of Pucher et $a l .{ }^{[12]}$ scholars. In summary, it is more reasonable and sensible to implement LC treatment as soon as possible for elderly patients with acute cholecystitis once the diagnosis is clear and proper preoperative preparations are perfected.

\section{TABLE 1: COMPARISON OF INTRAOPERATIVE CONDITIONS OF PATIENTS IN EACH GROUP}

\begin{tabular}{|c|c|c|c|c|}
\hline Group & & A Group $(n=32)$ & B Group $(n=32)$ & C Group $(n=32)$ \\
\hline \multirow{2}{*}{ Gender (example) } & male & 18 & 20 & 18 \\
\hline & Female & 14 & 12 & 14 \\
\hline Average age (y) & & $65.12 \pm 10.89$ & $65.26 \pm 10.59$ & $65.06 \pm 10.51$ \\
\hline BMI $\left(\mathrm{kg} / \mathrm{m}^{2}\right)$ & & $22.48 \pm 2.64$ & $22.49 \pm 2.03$ & $22.79 \pm 2.03$ \\
\hline \multirow{4}{*}{ Clinical manifestations } & bellyache & 31 & 28 & 29 \\
\hline & fever & 4 & 2 & 4 \\
\hline & sick and vomit & 8 & 12 & 8 \\
\hline & Yellow sclera & 5 & 3 & 2 \\
\hline \multirow{4}{*}{ Types of complications } & diabetes & 4 & 4 & 4 \\
\hline & Essential hypertension & 2 & 4 & 2 \\
\hline & $\begin{array}{l}\text { Chronic obstructive } \\
\text { pulmonary disease }\end{array}$ & 4 & 2 & 4 \\
\hline & other illnesses & 4 & 4 & 2 \\
\hline
\end{tabular}


TABLE 2: COMPARISON OF SURGICAL CONDITIONS OF PATIENTS IN EACH GROUP

\begin{tabular}{lccc}
\hline Group & $\begin{array}{c}\text { A Group } \\
(\mathrm{n}=32)\end{array}$ & $\begin{array}{c}\text { B Group } \\
(\mathrm{n}=32)\end{array}$ & $\begin{array}{c}\text { C Group } \\
(\mathrm{n}=32)\end{array}$ \\
\hline Operation time (min) & $45.12 \pm 4.02$ & $44.12 \pm 4.02$ & $65.26 \pm 3.15$ \\
Intraoperative blood loss (mL) & $62.48 \pm 6.21$ & $62.48 \pm 6.59$ & $98.45 \pm 6.45$ \\
Postoperative bowel function & $3.02 \pm 0.12$ & $3.04 \pm 0.23$ & $5.86 \pm 0.64$ \\
recovery time (d) & $6.48 \pm 0.56$ & $6.15 \pm 0.55$ & $10.25 \pm 1.23$ \\
Hospitalization time (d) & & \\
\hline
\end{tabular}

TABLE 3: COMPARISON OF POSTOPERATIVE COMPLICATIONS OF PATIENTS IN EACH GROUP [N (\%)]

\begin{tabular}{lccc}
\hline Group & $\begin{array}{c}\text { A Group } \\
(\mathrm{n}=32)\end{array}$ & $\begin{array}{c}\text { B Group } \\
(\mathrm{n}=32)\end{array}$ & $\begin{array}{c}\text { C Group } \\
(\mathrm{n}=32)\end{array}$ \\
\hline Bleeding & $0(0.00)$ & $2(6.25)$ & $0(0.00)$ \\
Lung infection & $2(6.25)$ & $2(6.25)$ & $2(6.25)$ \\
$\begin{array}{l}\text { Incision and abdominal cavity } \\
\text { infection }\end{array}$ & $2(6.25)$ & $0(0.00)$ & $0(0.00)$ \\
$\begin{array}{l}\text { Bile leakage and intestinal } \\
\text { fistula }\end{array}$ & $2(6.25)$ & $0(0.00)$ & $2(6.25)$ \\
$\begin{array}{l}\text { Deep vein thrombosis } \\
\text { Myocardial infarction }\end{array}$ & $0(0.00)$ & $0(0.00)$ & $2(6.25)$ \\
Total & $0(0.00)$ & $2(6.25)$ & $0(0.00)$ \\
\hline
\end{tabular}

\section{Author's contributions:}

Fei Guo and Anna Bao had contributed equally to this work.

\section{Conflict of interests:}

The authors declared no conflicts of interest.

\section{REFERENCES}

1. Gerard J, Luu MB, Poirier J, Deziel DJ. Acute cholecystitis: comparing clinical outcomes with TG13 severity and intended laparoscopic versus open cholecystectomy in difficult operative cases. Surg Endosc 2018;32(9):3943-8.

2. Qu JW, Xin C, Wang GY, Yuan ZQ, Li KW. Feasibility and safety of single-incision laparoscopic cholecystectomy versus conventional laparoscopic cholecystectomy in an ambulatory setting. Hepatobiliary Pancreat Dis Int 2019;18(3):273-7.

3. Rojas CL, Aguayo WG, Molina GA, Ochoa C, Neira A, Vinueza ME. Acute cholecystitis and residual choledocholithiasis in a situs inversus patient, successful laparoscopic approach and ERCP a case report from Ecuador. Ann Med Surg 2020;54:1015.

4. Navuluri R, Hoyer M, Osman M, Fergus J. Emergent treatment of acute cholangitis and acute cholecystitis. Semin Intervent Radiol 2020;37(1):14-23.

5. Shi H, Wan J, Xu SP, Liao L. A retrospective analysis of clinical characteristics and mortality risks in elderly patients with acute cholecystitis and cholangitis. Zhonghua Nei Ke Za Zhi 2019;58(6):415-8.

6. Chen S, Wu X, Zhang H, Guo F, Wang L, Bao A. Feasibility study of laparoscopic resection for acute gallbladder inflammation. Chin J Endosc Surg 2015;8(2):136-8.
7. Wennmacker SZ, Dijkgraaf MG, Westert GP, Drenth JP, van Laarhoven CJ, de Reuver PR. Persistent abdominal pain after laparoscopic cholecystectomy is associated with increased healthcare consumption and sick leave. Surgery 2018;163(4):661-6.

8. Umemura A, Suto T, Nakamura S, Fujiwara H, Endo F, Nitta $\mathrm{H}$, et al. Comparison of single-incision laparoscopic cholecystectomy versus needlescopic cholecystectomy: A single institutional randomized clinical trial. Dig Surg 2019;36(1):53-8.

9. Kowalewski KF, Garrow CR, Proctor T, Preukschas AA, Friedrich M, Müller PC, et al. LapTrain: multi-modality training curriculum for laparoscopic cholecystectomy - results of a randomized controlled trial. Surg Endosc 2018;32(9):38308.

10. Lockhart S, Singh-Ranger G. Rouviere's sulcus-Aspects of incorporating this valuable sign for laparoscopic cholecystectomy. Asian J Surg 2018;41(1):1-3.

11. Matsui Y, Satoi S, Hirooka S, Kosaka H, Kawaura T, Kitawaki T. Reappraisal of previously reported meta-analyses on antibiotic prophylaxis for low-risk laparoscopic cholecystectomy: an overview of systematic reviews. BMJ open 2018;8(3):28-40.

12. Pucher PH, Brunt LM, Davies N, Linsk A, Munshi A, Rodriguez HA, et al. Outcome trends and safety measures after 30 years of laparoscopic cholecystectomy: a systematic review and pooled data analysis. Surg Endosc 2018;32(5):2175-83.

This is an open access article distributed under the terms of the Creative Commons Attribution-NonCommercial-ShareAlike 3.0 License, which allows others to remix, tweak, and build upon the work non-commercially, as long as the author is credited and the new creations are licensed under the identical terms

This article was originally published in a special issue, "Therapeutic Perspectives in Biomedical Research and Pharmaceutical Sciences and their Nursing Methods" Indian J Pharm Sci 2021:83(4)Spl issue "1-4" 